\title{
DOMINANCE OF THE RESONANT MODES IN A HOMOGENEOUS DIELECTRIC SPHEROIDAL PARTICLE ILLUMINATED WITH A FOCUSED GAUSSAIN BEAM
}

\author{
Elsayed Esam M. Khaled \\ Elec. Eng. Dept., Faculty of Engineering, Assiut University \\ Esamk54_2000@hotmail.com
}

(Received June 29, 2009 Accepted September 1, 2009).

\begin{abstract}
Dominance of the resonant modes on the internal intensities of a homogeneous spheroidal particle illuminated with a focused, polarized, and shifted Gaussian beam is investigated. The results are calculated for a wider range of size parameter and axial ratio for both prolates and oblates spheroids. The plane wave spectrum and the T-matrix methods are used to calculate the internal electric field intensities. The results show that the resonant modes in an oblate spheroid are more pronounced than in a prolate spheroidal particle. The resonant modes in a spheroidal particle have lower quality factor than those in a spherical particle. The behavior of the internal intensities shows that a high quality factor (loworder) resonant mode(s) can dominate the internal intensities over a wide range of size parameter. This dominance of the resonant modes in a spheroidal particle is larger than those in a spherical particle.
\end{abstract}

\section{INTRODUCTION}

Light scattering by spheroidal particles are important in many areas such as characterization of chemical and biological substances [1], laser propagation through aerosols and dust particles [2], and investigation of nonlinear optical processes in droplets. These applications based on illuminating the particle by a plane wave or a shaped laser beam and, consequently, on the resultant field intensity distributions inside and outside the particle. These intensity distributions depend on the shape and frequency of the incident field, and on the shape and characteristics of the particle. Since the intensities distributions for resonant particles are different than those for offresonant particles then there is a substantial need to study behaviors of the resonant modes in spheroidal particles and the related internal intensity distributions.

The behaviors of the resonant modes in spheroidal particles have been studied previously for different shapes of an incident wave [3-5]. In the case of a Gaussian beam illumination the resonances are more easily excited when an incident beam is focused at or near the edge of a particle than when the beam is focused near the particle axis [5]. When the incident frequency is on a morphology-dependant resonances (MDR) of a particle the energy can be high. In spherical particles it had been shown that the high-Q resonant modes dominate the internal energy even when the incident frequency is hundreds of linewidths away from the resonance location [5]. This dominance of the high-Q modes depends on the size parameter of the particle and on the beam position with respect to the particle. For nonspherical particles the MDRs are different than those of spherical particles. Although the field intensity distributions for 
spheroidal particles illuminated with a plane wave or focused Gaussian beam are computed previously [6-12] no studies have been published in the literature describing the behaviors of the internal field intensity inside a spheroidal particle at or near resonance. Also effects of the shape of a spheroidal particle on dominance of the resonant modes are not investigated. The indication of the magnitude of the internal intensities distributions in a resonant mode and how it depends on the shape and frequency of the incident wave are important to understand nonlinear optical processes in spheroidal particles.

Nonlinear optical processes such as stimulated Raman scattering [13], stimulated Brillouin scattering [14], third-order sum-frequency generation [15], lasing [16], and coherent anti-stokes Raman scattering (CARS) [17] are much affected by resonant modes in spherical particles. Such types of processes can be generated in a spherical particle using a focused laser beam illuminated at or closer to the particle's surface. Moreover these nonlinear optical processes occur with relatively low levels of pump power. A main reason for low powers required is that the MDRs of the particle provide feedback for the output fields [3]. Near or at the MDRs the transition rates of molecules and the fields at the pump frequencies inside the droplets are enhanced [4]. Some of these MDRs have high computed quality factors Q. These high-Q MDRs reduce the thresholds of the nonlinear optical processes in spherical particles [3]. Also the output power levels of nonlinear optical processes depend on the overlap integrals of the incident fields and the resultant internal field distributions. Consequently in resonant spherical particles the high-Q output modes dominate the output fields because they have the best spatial overlaps with the incident (pump) fields [5]. The internal field distributions depend mainly on the particle shape, and on the spatial distribution and frequency of the incident field. Therefore it is important to study the behaviors of the internal field distributions for a shaped particle (such as spheroidal particle) at or near resonances.

In this paper effects of the particle's shape on the behavior of the resonant modes are investigated. Dominance of the resonant modes on the internal intensities of a spheroidal particle is also described. The results are calculated for a wider range of size parameter and axial ratio for both prolates and oblates spheroids. The theoretical analysis is outlined in Section 2. Results and discussions are given in Section 3. Section 4 contains the Conclusions.

\section{THEORETICAL AND NUMERICAL ANALYSIS}

Figure 1 shows a homogeneous spheroidal particle of a refractive index $m$ centered at the origin of a right-handed Cartesian coordinate system $(x, y, z)$. A spherical coordinate system $(r, \theta, \varphi)$ is also shown. The refractive index of the surrounding media is unity. The radius of the spheroid along the $x$-axis is $b$ and that along the $z$-axis is $a$. The spheroid is oriented with respect to the Cartesian coordinate system such that its semimajor axis dimension is along the $\mathrm{x}$-axis (oblate case) or along the $\mathrm{z}$-axis (prolate case). The axial ratio is $\rho=a / b(\rho<1$ for oblate case and $\rho>1$ for prolate case). The particle is illuminated with a lowest order $\left(\mathrm{TEM}_{\mathrm{oo}}\right)$ monochromatic Gaussian beam (or other shapes) polarized in the $x z$-plane and of a wavelength $\lambda$. The incident beam propagates in the $z$-direction. The minimum spot size of the beam is $\omega_{0}$. The focal point of the beam is located at an arbitrary point $\left(x_{o}, y_{o}, z_{o}\right)$. 


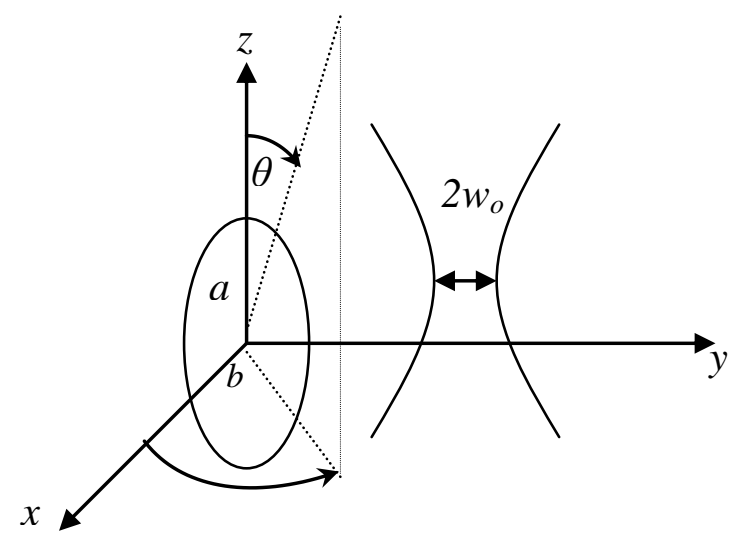

Fig. 1. A spheroidal particle centered at the origin of a Cartesian coordinate system $(x, y, z)$. The radii $a$, and $b$ of the particle are along the $z$-and $x$-axis respectively. The particle illuminated with a focused shifted Gaussian beam of a minimum spot size $w_{o}$.

The incident Gaussian beam can be expressed in Vector Spherical Harmonics (VSH) as[18],

$$
E^{i n c}(k r)=P^{2} \sum_{m} \sum_{n} D_{m n}\left[a_{e m n}^{t} M_{e m n}^{1}(k r)+a_{o m n}^{t} M_{o m n}^{1}(k r)+b_{e m n}^{t} N_{e m n}^{1}(k r)+b_{o m n}^{t} N_{o m n}^{1}(k r)\right] \text {, }
$$

where $P$, and $D_{m n}$ are normalization factors. The $m$ and $n$ are integer numbers denoting to the azimuthal mode number and the mode index respectively. The vectors $M^{l}(k r)$, and $N^{l}(k r)$ are the VSH of the first kind. The letters $e$ and $o$ stand for even and odd modes respectively. The coefficients $a_{\text {emn }}^{t}, a_{\text {omn }}^{t}, b_{e m n}^{t}$, and $b_{\text {omn }}^{t}$ are the expansion coefficients of the incident field. The method can be applied to model any physically realizable beam which can be expressed as a sum of homogeneous plane waves. Also the technique can be used to model a beam that has no analytical representation such as a laboratory non-exact Gaussian beam which generated, in general, with a reducing oscillating tail.

The internal electric fields $E^{\text {int }}$ of a homogeneous dielectric particle illuminated with a Gaussian beam is [8],

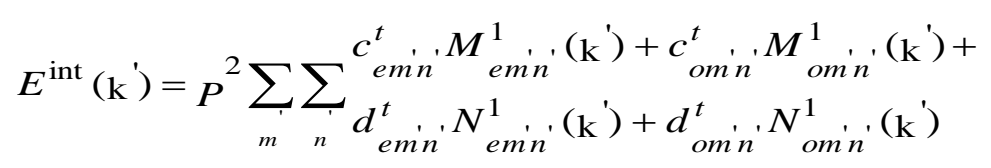

where $k^{\prime}=r k \sqrt{ } \varepsilon_{r} ; \varepsilon_{r}$ is the complex dielectric constant of the scatterer relative to that of the surrounding medium. The $c^{t}{ }_{e m^{\prime} n^{\prime}} c^{t}{ }_{o m^{\prime} n^{\prime}} d^{t}{ }_{e m^{\prime} n^{\prime}}$ and $d^{t}{ }_{o m^{\prime} n^{\prime}}$ are the expansion coefficients of the internal electric field. In case of an axisymmetric object $m^{\prime}=m$. The internal field expansion coefficients for each azimuthal mode, $m$, can be calculated from the incident field expansion coefficients using the T-matrix method. All mathematical analysis can be found in Reference [12]. 


\section{RESULTS AND DISCUSSION}

To investigate the effects of the shape of a particle on the behavior of the internal intensities we consider a homogeneous spherical particle of an axial ratio $\rho=a / b=1$ and a refractive index $m=1.36$. The size parameter of the particle is $x=47.3094299$ which corresponding to the $T E_{58,1}$ resonant mode (MDR). The particle is illuminated with a focused Gaussian beam of a wavelength $\lambda=1.064 \mu \mathrm{m}$ and a minimum spot size $w_{o}=2 \mu \mathrm{m}$ propagating in the z-direction. The focal point of the beam is shifted to $x_{o}=11.098098$, $y_{o}=0, z_{o}=0$. Summation of magnitudes of the internal field expansion coefficients that is $\sum_{m} \sum_{n} n^{\prime}\left|c_{e m n}^{t}\right|+\left|c_{o m n}^{t}\right|+\left|d^{t}{ }_{e m n}\right|+\left|d_{\text {omn }}^{t}\right|$ are computed as a function of the axial ratio $\rho$. The reasons for calculating summation of magnitudes of the expansion coefficients are: first they do not depend on locations inside or outside the particle, second each certain coefficient $n$ ' has maximum contribution to the corresponding resonant mode. Therefore summation of magnitudes of the expansion coefficients contains peaks at corresponding resonant modes. Since the spherical particle is at the resonant mode $T E_{58,1}$ then the summation will show maximum value at the size parameter 47.3094299 for $\rho=1$ (spherical particle) case. The previously considered spherical particle is then deformed by gradually increasing its axial ratio $\rho$ (prolate case) and then gradually decreasing $\rho$ (oblate case). The size parameter of the spheroid at each step is calculated using the equivalent spherical volume concept that is $x_{\text {spheroid }}=$ $x_{\text {sphere }}(a / b)^{2 / 3}$. The computed results are shown in Fig. 2. The results show that the resonant modes in the oblate spheroidal particle case are more pronounced than in the prolate spheroidal particle case. Also the quality factors of the modes in spheroidal particle are much less than that in the spherical particle. Moreover the resonant mode in spherical particle is much sensitive to the particle deformation than those in spheroidal particle.

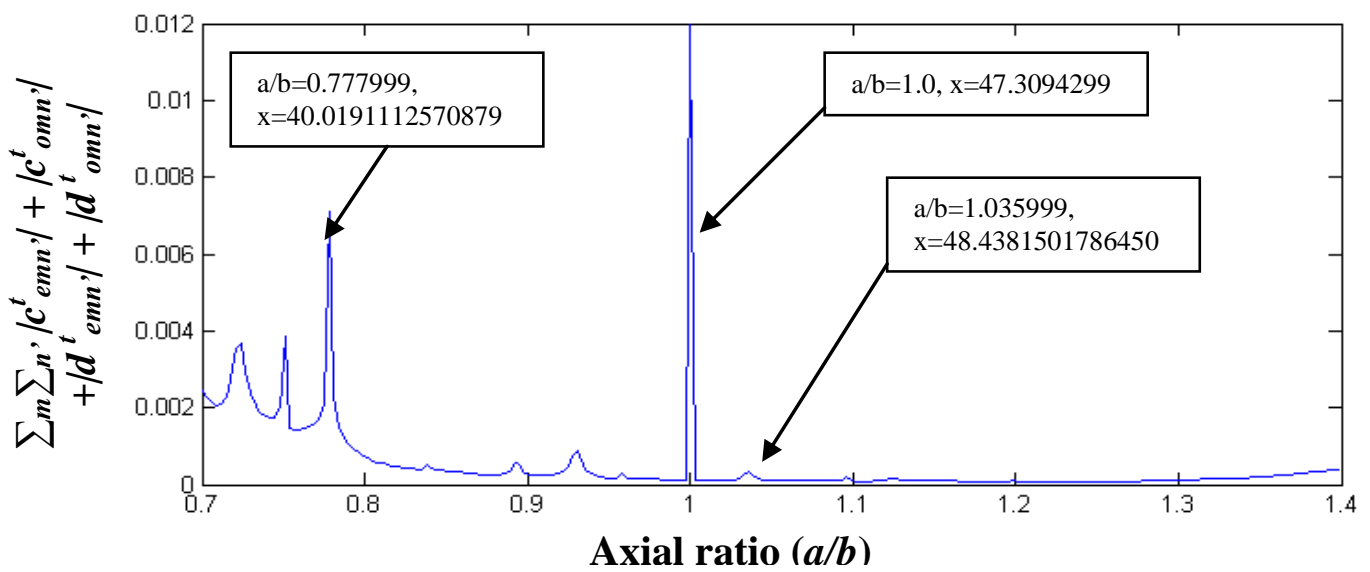

Fig. 2 Summation of magnitudes of the internal field expansion coefficients $\sum_{m} \sum_{n}$, $\left|c_{\text {emn }}^{t}\right|+\left|c_{\text {omn }}^{t}\right|+\left|d_{\text {emn }}^{t}\right|+\left|d_{\text {omn }}^{t}\right|$, as a function of axial ratio $\rho=a / b$ of a spheroidal particle of refractive index $\mathrm{m}=1.36$ and size parameter 47.3094299 which is corresponding to $T E_{58,1}$ mode when $\rho=1$ (spherical particle case).

The particle is illuminated with an off-axis Gaussian beam of a wavelength $\lambda=1.064 \mu \mathrm{m}$ and minimum spot size $w_{o}=2 \mu \mathrm{m}$. The beam propagates in $z$-direction and is shifted to $x_{o}=11.098098 \mu m, y_{o}=z_{o}=0$. 
Summation of magnitudes of the internal field expansion coefficients as a function size parameter of a spheroidal particle is computed to study dominance of the resonant modes on the internal intensities. First the summation is calculated for an oblate spheroidal particle of a refractive index $\mathrm{m}=1.36$ and an axial ratio $\rho=0.777999$. The particle is illuminated with an off-axis Gaussian beam of parameters as those in Fig. 2. The calculated results are shown in Fig. 3.

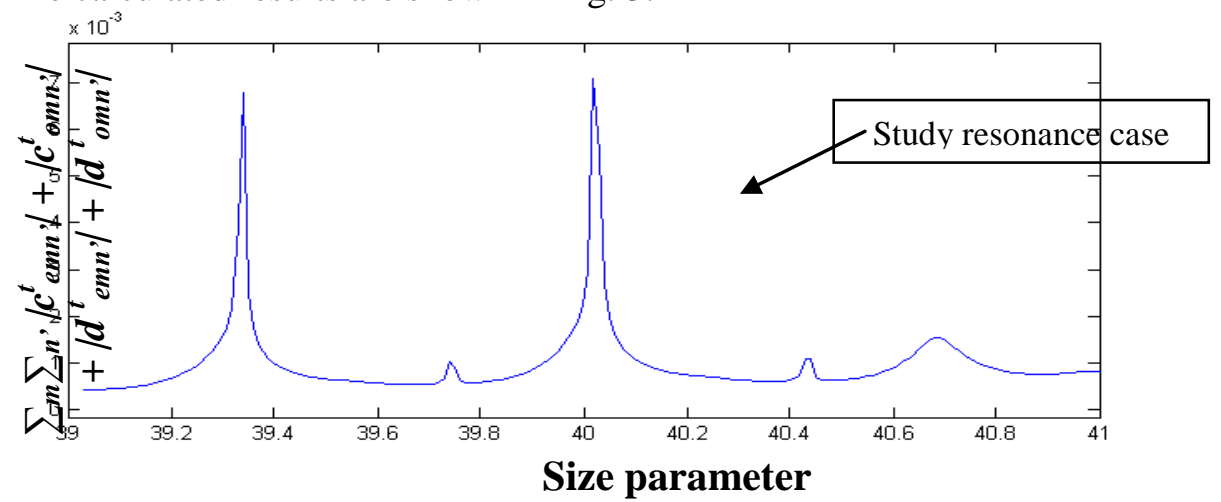

Fig. 3 Summation of magnitudes of the internal field expansion coefficients $\sum_{m} \sum_{n}$ ' $\left|c_{\text {emn }}^{t}\right|+\left|c_{\text {omn }}^{t}\right|+\left|d_{\text {emn }}^{t}\right|+\left|d_{\text {omn }}^{t}\right|$, as a function of of size parameter of an oblate spheroidal particle of refractive index $\mathrm{m}=1.36$ and an axial ratio $\rho=0.777999$. The oblate is illuminated with an off axis Gaussian beam of parameters as those of Fig. 2.

Figure 3 demonstrates that there is a distinct mode at size parameter 40.01911126 which is (with equivalent spherical volume concept) equal to the spherical size parameter 42.3094299 . This size parameter is corresponding to $T E_{58,1}$ MDR in the spherical particle case $(\rho=1)$. Since there are no definitions for the resonant modes in a spheroidal particle the resonant mode occurs at $\mathrm{x}=40.01911126$ can not be defined as the $\mathrm{TE}_{58,1}$ resonant mode. It could be another mode. Also there are other distinct modes at different size parameters. Some of these pronounced modes are high-Q modes which suggested that low-order modes can be excited in an elongated oblate spheroidal particle. Also the results show that the high-Q modes dominate the internal intensities for a wide range of size parameter. To define number of linewidths that a mode is dominated we need to know the width of the resonant mode, $\Delta \mathrm{x}$, in a spheroidal particle which is not defined or investigated in the literature.

For more investigation of the dominance of the low-order mode we calculated summation of magnitudes of the expansion coefficients corresponding to $\mathrm{n}=58$ and $\mathrm{n}^{\prime}=50$ over the azimuthal modes $m$, that is $\sum_{m}\left|c_{\text {em5 }}^{t}\right|+\left|c_{\text {om58 }}^{t}\right|$ and $\sum_{m}\left|c_{\text {em5o }}^{t}\right|+\left|c_{\text {om5 }}^{t}\right|$ (the $c_{m n}^{t}$, coefficients are corresponding to the transverse electric, TE, modes whereas $d$ ${ }_{m n}^{t}$, coefficients are corresponding to the transverse magnetic, TM, modes [3]). The summation is computed as a function of size parameter $\mathrm{x}$ for the same case as that in Fig. 3. The results are demonstrated in Fig. 4. The results clarify that the resonant modes in this case dominate the internal intensities over a wide range of size parameter (many linewidths) away from resonance locations. 


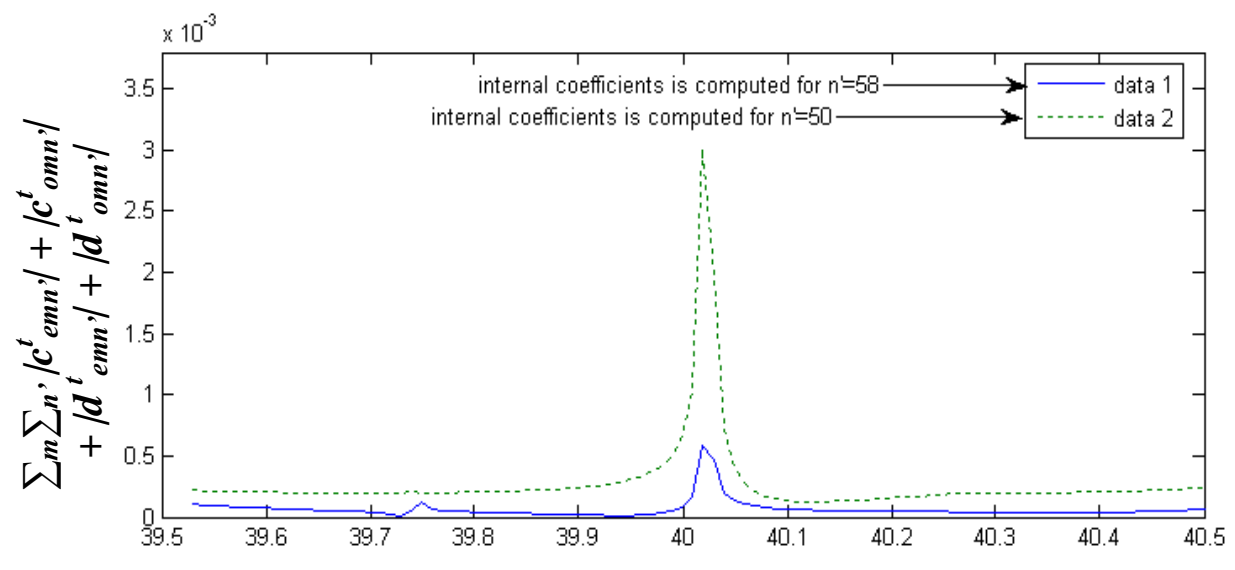

Size parameter

Fig. 4 Summation of magnitudes of the internal field expansion coefficients $\sum_{m^{\prime}}\left|c_{e m 58}^{t}\right|$ $+\left|c_{\text {om } 58}^{t}\right|$, and $\sum_{m^{\prime}}\left|c_{\text {em5o }}^{t}\right|+\left|c_{\text {om5 }}^{t}\right|$ as a function of of size parameter of an oblate spheroidal particle of refractive index $\mathrm{m}=1.36$ and an axial ratio $\rho=0.777999$. The oblate is illuminated with an off-axis Gaussian beam of parameters as those of Fig. 2.

We repeated the computation of the case in figure 3 except that the focal point of the beam is located at the origin that is on-axis illumination $\left(x_{o}=y_{o}=z_{o}=0\right)$. The computed results illustrated in Fig. 5. No resonant modes are excited. The on-axis Gaussian beam illumination does not excite resonant modes in an oblate spheroidal particle for any size parameter or axial ratio [11,12].

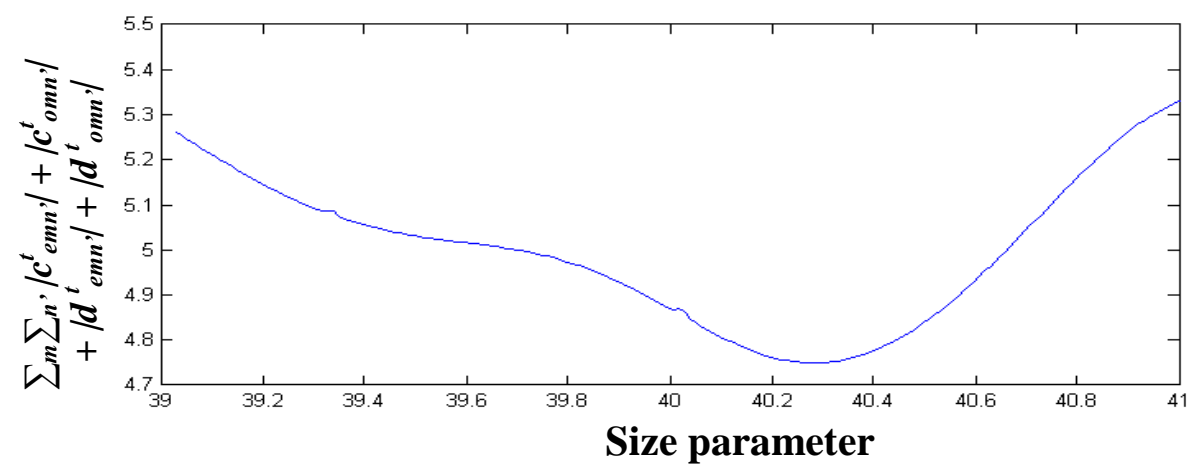

Fig. 5 Summation of magnitudes of the internal field expansion coefficients $\sum_{m} \sum_{n}$, $\left|c_{\text {emn }}^{t}\right|+\left|c_{\text {omn }}^{t}\right|+\left|d^{t}{ }_{\text {emn }},\right|+\left|d^{t}{ }_{\text {omn }}\right|$ as a function of of size parameter of an oblate spheroidal particle illuminated with an on-axis Gaussian beam. All the parameters are the same as those of Fig. 3 except that the focal point of the beam is located at the origin.

Summation of magnitudes of the internal field expansion coefficients is computed as a function of size parameter for a prolate spheroidal particle. The axial ratio of the particle is $\rho=1.035999$ and its refractive index is 1.36 . The particle is illuminated with an off-axis Gaussian beam of parameters as those of the case in Fig. 2. The calculated results are shown in Fig. 6. The on-axis Gaussian beam illumination 
case is also computed and illustrated in Fig. 7. The reason for choosing the axial ratio of the prolate $\rho=1.03599$ which is close to unity (a prolate of small elongation) is that as the axial ratio increases, i.e. elongated prolate the resonant modes diminished and disappeared as shown in Fig. 2. Figure 6 shows that some of the resonant modes have high $\mathrm{Q}$ and dominate the internal intensities over a wider range of size parameter than other modes. Also definitions for resonant modes and their linwidths are required to count number of linewidths that a resonant mode can dominate. These definitions are important to study nonlinear optical processes in spheroidal particles. That issue be a subject for future work. Figure 7 illustrated that, as in the case of the oblate spheroid, no resonant modes can be excited in a prolate spheroidal particle illuminated with an on-axis Gaussian beam.

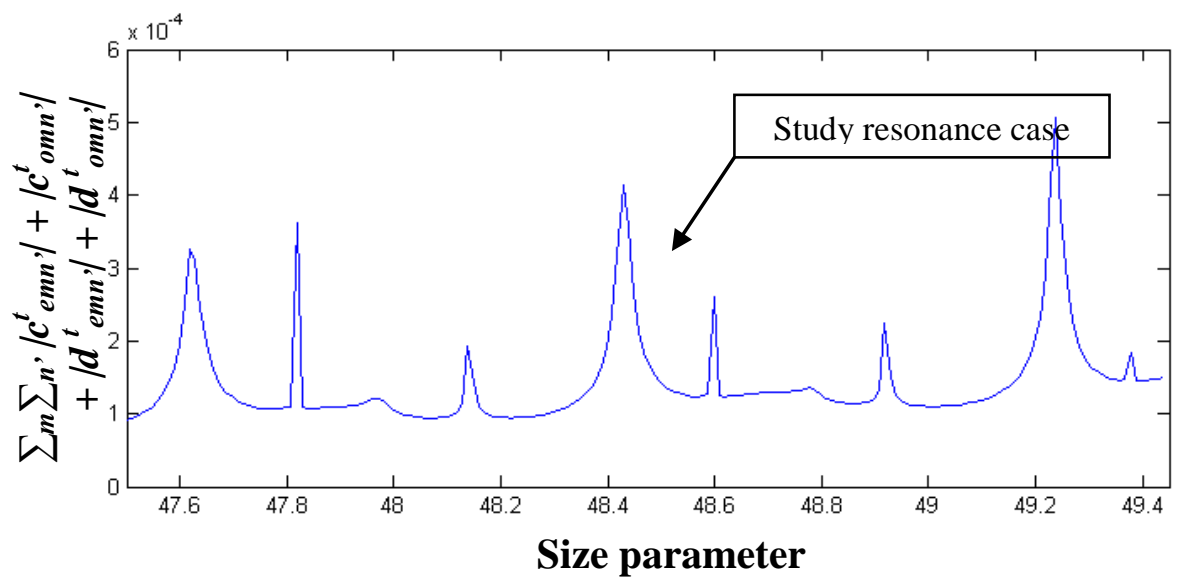

Fig. 6 Summation of magnitudes of the internal field expansion coefficients $\sum_{m} \sum_{n}$, $\left|c_{\text {emn }}^{t}\right|+\left|c_{\text {omn }}^{t}\right|+\left|d_{\text {emn }}^{t}\right|+\left|d_{\text {omn }}^{t}\right|$ as a function of of size parameter of a prolate spheroidal particle of refractive index $\mathrm{m}=1.36$ and an axial ratio $\rho=0.777999$. The oblate is illuminated with an off-axis Gaussian beam of parameters as those of Fig. 2.

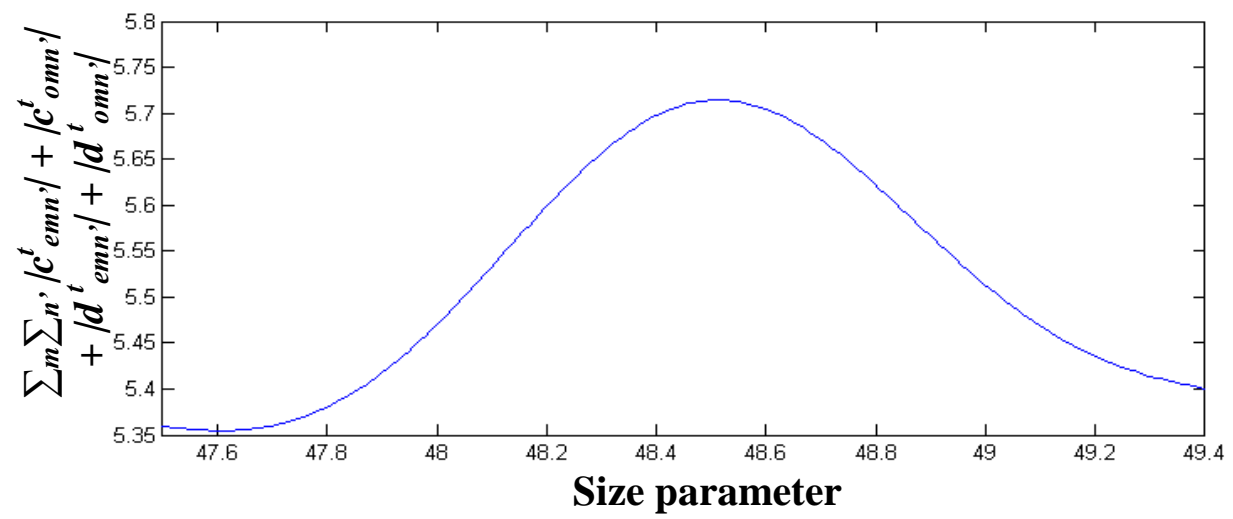

Fig. 7 Summation of magnitudes of the internal field expansion coefficients $\sum_{m} \sum_{n}$, $\left|c_{\text {emn }}^{t}\right|+\left|c_{\text {omn }}^{t}\right|+\left|d_{\text {emn }}^{t}\right|+\left|d_{\text {omn }}^{t}\right|$ as a function of of size parameter of an oblate spheroidal particle illuminated with an on-axis Gaussian beam. All parameters are the same as those of Fig. 6 except that the focal point of the beam is located at the origin. 
Fortran codes are built up to model incident Gaussian beams and to compute the field intensities inside and outside the spheroidal particle. Once the T-matrix for a certain particle is computed it can be used for different illuminations of each case. For all the previous cases we use a PC Pentium IV with $1.7 \mathrm{GHz}, 128 \mathrm{MB}$ Ram. The codes are not too much time consuming for computations. For example the real time for one job running on the machine to get results of the case in Fig. 2 is approximately 7 hours and 20 minutes for 200 points of calculations.

\section{CONCLUSIONS}

Behaviors of the resonant modes and their dominance on the internal intensities of a spheroidal particle illuminated with a focused, polarized, and shifted Gaussian beam are investigated. The results are calculated for a wider range of size parameter and axial ratio for both prolates and oblates spheroids. The plane wave spectrum and the Tmatrix methods are used to calculate the internal electric field intensities. The results show that a high quality factor (low-order) resonant mode(s) can dominate the internal intensities in either a prolate or an oblate spheroidal particle. This dominance in a spheroidal particle is less than that in a spherical particle.

Definitions and differentiations of the resonant modes and their linewidths in a spheroidal particle are important to determine dominance of the mode and to study nonlinear optical processes. This could be a subject for future work.

\section{REFERENCES}

[1] S. Asano, and G. Yamamoto, "Light scattering by a spheroidal particle", Appl. Opt., 14, 29-49, 1975.

[2] S. C. Hill, A. C. Hill, and P. W. Barber, "Light scattering by size/shape distributions of soil particles and spheroids", Appl. Opt., 23, 1025-1031, 1984.

[3] S. C. Hill and R. E. Benner, "Morphology-dependent resonances", in Optical Effects Associated with Small Particles, P. W. Barber and R. K. Chang, eds., World Scientific, Singapore, 3-61, 1988.

[4] H. Chew, "Radiation lifetimes of atoms inside dielectric particles", Phys. Rev. A 38, 3410-3416, 1988.

[5] E. E. M. Khaled, S. C. Hill, and P. W. Barber, "Internal electric energy in a spherical particle illuminated with a plane wave or off-axis Gaussian beam", Appl. Opt. 33, 524-532, 1994.

[6] D-S. Wang and P. W. Barber, "Scattering by inhomogeneous nonspherical objects", Appl. Opt., 18, 1190, 1979.

[7] A. Coletti, "Light scattering by nonspherical particles: a laboratory study", Aerosol Science and Technology, 3, 39, 1984.

[8] P. W. Barber, and S. C. Hill, Light Scattering by Particles: Computational Methods, World Scientific, Singapore, 1990.

[9] J. P. Barton and D. R. Alexander, "Electromagnetic fields for an irregularly shaped, near-spherical particle illuminated by a focused laser beam", J. Appl. Phys., 69, 7973, 1991. 
[10] J. P. Barton, "Internal and near-surface electromagnetic fields for a spheroidal particle with arbitrary illumination", Appl. Opt. 34, 5542-5551, 1995.

[11] E. E. M. Khaled, "Scattering of an on-axis arbitrarily-shaped focused electromagnetic beam by an elongated spheroidal object", Proceeding of the twenty-sixth national radio science conference (NRSC'26, 2009) sponsored by URSI and IEEE Egypt section, Faculty of Engineering, Future University, Cairo, Egypt, March. 17-19, 2009.

[12] E. E. M. Khaled, and H. Louka, " Scattered and Internal Intensities of a Homogeneous Dielectric Spheroidal Object Illuminated with an Arbitrary Focused Off-Axis Electromagnetic Beam", Journal of Engineering Sciences, Engineering Faculty, Assiut University, JES, Vol. 37, No. 4, July 2009.

[13] G. Chen, W. Acker, R. K. Chang, and S. C. Hill, "Fine structures in the stimulated Raman scattering from single droplets", Opt. Lett. 16, 117-119, 1991.

[14] J.-C. Zhang and R. K. Chang, "Generation and suppression of stimulated Brillouin scattering in single liquid droplets", J. Opt. Soc. Am B6, 151-153, 1989.

[15] S. C. Hill, D. H. Leach, and R. K. Chang, "Third-order sum frequency generation in droplets: model with numerical results for third-harmonic generation", J. Opt. Soc. Am. B 10, 16-33, 1993.

[16] J. D. Eversole, H-B. Lin, and A. J. Campillo, "Cavity-mode identification of fluorescence and lasing in dye-doped micro-droplets", Appl. Opt. 31, 1982-1991, 1992.

[17] J. Cooney and A. Gross, "Coherent anti-Stokes Raman scattering by droplets in the Mie size range", Opt. Lett., 7(5), 218, 1982.

[18] E. E. M. Khaled, S. C. Hill, and P. W. Barber, "Scattered and internal intensity of a sphere illuminated with a Gaussian beam", IEEE Trans., Antennas Propag., Ap-41, 295-303, 1993. 


\section{سيطرة موجات الرنين في جسيم من مادة عازلة متجانس وييضاوي \\ مسلط عليه شعاع جاوسين مركز \\ السيد عصام محمد خالد \\ قسم الهنسة الكهربائية ـ كلية الهندسة - جامعة أسيوط \\ Esamk54_2000@hotmail.com}

تم دراسة وفحص سيطرة شدة موجات الرنين على شدة المجالات الكهرومغناطيسية الكلية داخل جسيم

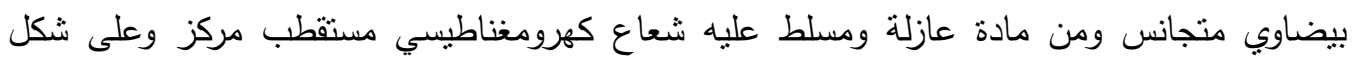

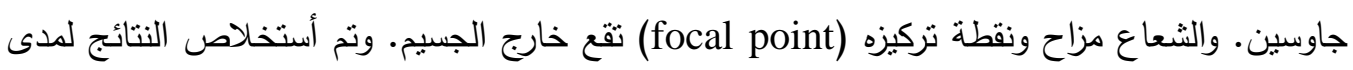
واسع من المعامل الحجمي (size parameter) ومعامل الأسنطالة (axial ratio) لكلا نوعي الجسيم البيضاوي وهما: الأول الجسيم مسنطال في أتجاه موازي لأتجاه انتشار الثشعاع (prolate) والثاني

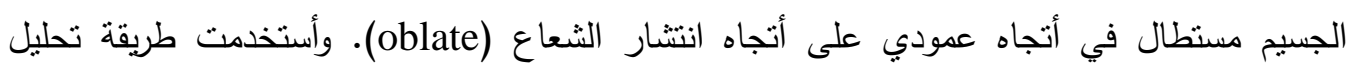
الثعاع لموجات مسنوية لتنثيل الثعاع وطريقة T-matrix لحساب شدة المجالات داخل الجسيم.

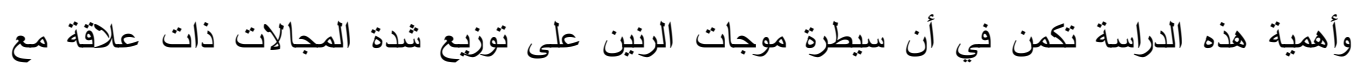
عمليات تفاعل الضوء الغير خطية مع الجسيمات وتطبيقاتها المختلفة.

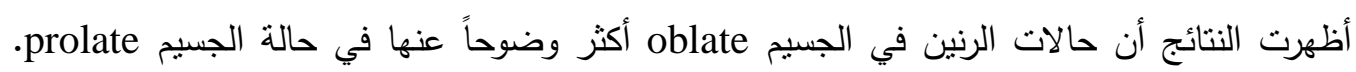

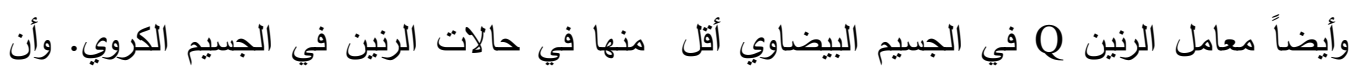

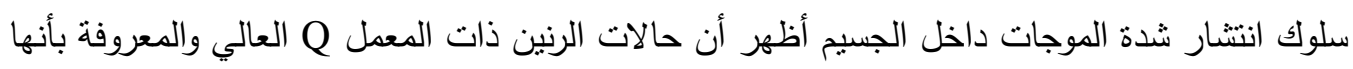
ذات الرتبة الأولى low-order modes نسيطر على نوزيع شدة المجالات لمدى كبير من المعامل الحجمي. هذه السيطرة في حالة الجسيم البيضاوي أكبر منها في حالة الجسيم الكروي. وخلصت الدراسة بأهية تحديد أسماء وقياس حالات الرنين في الأجسام البيضاوية التي تتكون من مواد التهاد

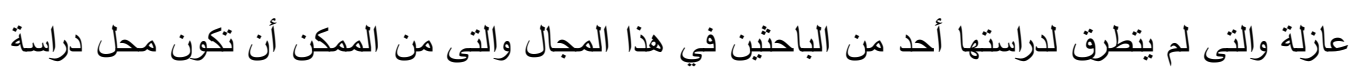
في المستقبل. 